\title{
Room-temperature broadband quasistatic magnetic cloak
}

\author{
Wei Jiang ${ }^{1}$, Yungui $\mathrm{Ma}^{1}$, Jianfei Zhu${ }^{1}$, Ge Yin ${ }^{1}$, Yichao $\mathrm{Liu}^{1}$, Jun Yuan ${ }^{1}$ and Sailing $\mathrm{He}^{1,2}$ \\ In the past decade, invisible cloaks have experienced rapid research development in the metamaterial community driven by their \\ revolutionary practical potentials. Among them, magnetic cloaks, which are able to conceal metallic or magnetic objects from \\ electromagnetic induction detection, have attracted a great amount of attention. However, applications of these reported devices \\ are limited by their low-temperature environment requirement because of the involvement of superconductors to acquire the \\ perfect diamagnetic response. In this work, we remove this temperature hurdle by fully taking the diamagnetic features of usual \\ metals and demonstrate a three-dimensional room temperature quasistatic magnetic cloak using a ferromagnetic metallic bilayer \\ structure. Experimentally, our device exhibits a prominent cloaking effect in a wide frequency range from 5 to $250 \mathrm{kHz}$ with a \\ maximum field disturbance ratio $<0.5 \%$. The practical potential is verified through a commercial handheld metal probe working \\ at $25 \mathrm{kHz}$. Our results unambiguously show that an invisible cloak may be realized in the low-frequency region for scenarios \\ where screening an external magnetic field without disturbance is specifically demanded.
}

NPG Asia Materials (2017) 9, e341; doi:10.1038/am.2016.197; published online 13 January 2017

\section{INTRODUCTION}

In the past decade, invisible cloaking has become a major research topic in the field of metamaterials and has inspired enthusiastic research across multiple disciplines. ${ }^{1-7}$ However, the outstanding theoretical performance of invisible cloaks in manipulating electromagnetic (EM) waves is critically challenged in reality by the complex material properties demanded from the general designs applying transformation optics. It is not easy to attain simultaneously both magnetic and electric responses in predetermined ways even by using metamaterials, although different cloaking approaches with reduced parametric requirements have been proposed or partially implemented..$^{8-15}$ Additionally, it is of practical and conceptual importance for invisible cloaking to perform at low or zero frequencies, ${ }^{16-24}$ in particular for magnetic fields, which are ubiquitous in modern technologies and our daily lives. In 2012, Gömöry et al. ${ }^{17}$ and Narayama et al. ${ }^{18}$ independently reported the first experimental demonstrations of successfully hiding objects in static magnetic fields using a bilayer approach and a traditional transformation optics design, respectively. ${ }^{17,18}$ The efficiency of the bilayer approach proposed by the first group was quickly verified and applied to other Laplace equations governing the evolutions of different physical phenomena. ${ }^{25-31}$ In practice, a magnetic cloak that is able to perform under alternating currents will be more meaningful, as one usually relies on EM induction to uncover hidden metallic or magnetic objects. The same group extended their original two-dimensional bilayer design to A.C. fields and indicated a technical possibility within a frequency range of several hundred Hertz. ${ }^{21,22}$ We recently extended the bilayer approach and demonstrated a three-dimensional magnetic cloak working from DC to $250 \mathrm{kHz}$, which covered nearly all of the EM induction operational bands. ${ }^{24}$ In our design, a single-crystal superconductor (SC) shell was used as the key component to perfectly shield magnetic fields, whereas a ferromagnetic (FM) outer shell was prepared to balance out the inner diamagnetic response. This conceptual accomplishment has limited significance in practice, as a liquid-nitrogen temperature environment is an absolute requirement for any device involving SCs. Progress has been achieved by using non-superconducting magnetic metamaterials to enhance wireless power transmission ${ }^{32}$. In this work, we attempt to remove this hurdle in a magnetic cloak and unambiguously show the technical possibility of room-temperature broadband three-dimensional magnetic cloaking effect by using a proper combination of normally available materials. Our results indicate that a practical invisible cloak may be realized at last at low frequencies from several to hundreds of kilohertz for scenarios where perfect anti-EM induction detection is required.

\section{MATERIALS AND METHODS}

Simulation

We used the axial-symmetrical module in the RF domain by the Comsol Multiphysics Software (COMSOL, Shanghai, China) to simulate the dynamic

\footnotetext{
${ }^{1}$ State Key Lab of Modern Optical Instrumentation, Centre for Optical and Electromagnetic Research, College of Optical Science and Engineering, Zhejiang University, Hangzhou City, China and ${ }^{2}$ Department of Electromagnetic Engineering, School of Electrical Engineering, Royal Institute of Technology, Stockholm, Sweden Correspondence: Professor Y Ma, State Key Lab of Modern Optical Instrumentation, Center for Optical and Electromagnetic Research, Zhejiang University, College of Optical Science and Engineering, Zhejiang University, Zijin'gang Campus, Room 209, East building 5, Hangzhou City 310058, China.

E-mail: yungui@zju.edu.cn

or Professor S He, Department of Electromagnetic Engineering, School of Electrical Engineering, Royal Institute of Technology, Stockholm 100 44, Sweden. E-mail: sailing@kth

Received 26 September 2016; revised 2 November 2016; accepted 4 November 2016
} 
a

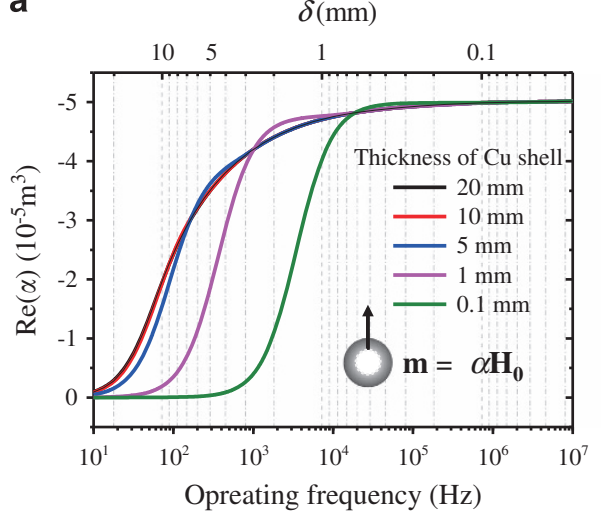

b

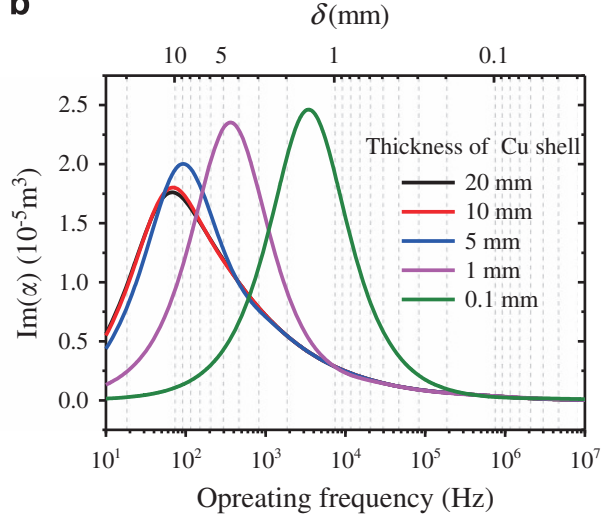

Figure 1 Effective magnetic polarizability of a spherical $\mathrm{Cu}$ shell. Real (a) and imaginary (b) parts at various frequencies. The top $x$ axis represents the penetration depth $\delta$ of waves in copper. The Cu shell has a fixed outer radius of $20 \mathrm{~mm}$ and five different thicknesses (20,10,5,1 and $0.1 \mathrm{~mm})$. The inset in (a) depicts the magnetic dipole and defines the effective polarizability $\alpha$ of the copper shell.

magnetic behaviors of the samples. A cylindrical current surface surrounding the spherical samples was defined to excite the homogeneous incident magnetic field. The direction of the magnetic field was along the $z$ axis of the cylindrical coordinate. The diameter of this cylindrical surface was far larger than that of the samples. All of these structures were enclosed by an air volume with the outermost edges set by the scattering boundary condition. To evaluate the magnetic response of the pure spherical copper shell, we first collected the magnetic field along a semicircle (of radius $=40 \mathrm{~cm}$ ) covering the shell and fitted it with the angular distribution function of the field of a standard magnetic dipole, through which we obtained the effective magnetic polarizability $\alpha$ of the copper shell.

\section{Sample fabrication}

The ferrite shell was a mixture of NiZn ferrite powders and paraffin matrix with the composition optimized through changing the volume ratio. The optimized permeability remained at a stable value $\mu=1.62$ for an external magnetic field $<20 \mathrm{mT}$. The copper shell was mechanically manufactured.

\section{Sample characterization}

To check the response in the homogeneous external magnetic field, the samples were placed at the central position of a pair of custom-made Helmholtz coils (diameter $=33 \mathrm{~cm}$, spacing $=11 \mathrm{~cm}$ and turn $=30$ ). A signal generator (Standard DS345, Stanford Research Systems Inc., Sunnyvale, CA, USA) was used to excite the field through the coils, and the $z$-component oscillating magnetic field near the sample was inductively measured by a lock-in amplifier (Signal Recovery 7270, AMETEK Inc., Santiago, CA, USA) through a 4-mm-diameter coil prober made of 80-turn copper wire loops. A handheld commercial metal scanner (Tianxu TX1001B, Tianxun Electronic Equipment Co., Ltd., Shanghai, China) was used to show the practical potential of the device perceiving the near-field metallic signal. The scanner creates alerts by 'beeping' and showing red flashes when a metal is detected. An oscilloscope (Agilent 34410A, Agilent Technologies Inc., Beijing, China) was used to give the exact measurement of the signal perceived by the scanner.

\section{RESULTS}

\section{Design of the device}

For an ideal SC, the flux exclusion property can be alternatively realized by a good conducting metal at dynamic frequencies by its perfect magnetic field shielding capability because of the huge conductivity $\sigma$ corresponding to a small penetration depth $\delta(=\sqrt{1 / \pi f \mu \sigma}$, where $f$ is frequency and $\mu$ is permeability) and small resistive loss. ${ }^{16}$ If one does not place particular concern on zero and ultra-low frequencies (e.g., $f<1 \mathrm{kHz}$ ), it is technically possible to achieve similar shielding and diamagnetic properties using normal metals at relatively high frequencies. For example, copper, which is used in this work, has $\delta=0.9 \mathrm{~mm}$ at $5 \mathrm{kHz}$ and in terms of the bilayer approach may replace SC as the inner diamagnetic shell at a thickness $t_{\mathrm{m}}>0.9 \mathrm{~mm}$ for frequencies above $5 \mathrm{kHz}$, which interestingly covers the working bands of most small-depth metal detectors. To do this, we first need to evaluate the behavior of a spherical metal shell in the excitation of a uniform external magnetic field $\mathbf{H}_{0}$. Under the quasistatic condition, the spherical metal shell will mimic a magnetic dipole with momentum $\mathbf{m}$ perceived from the far field. ${ }^{33}$ Figures $1 \mathrm{a}$ and $\mathrm{b}$ show the variation of the real and imaginary parts of the magnetic polarizability of the spherical $\mathrm{Cu}$ shell with frequency at different $t_{\mathrm{m}}$ with the outer radius fixed at $20 \mathrm{~mm}$. Here the magnetic polarizability of the metallic shell $\alpha(\omega)$ is numerically fitted from the perturbed magnetic fields distributed along a semicircle line obtained by a COMSOL model simulation rather than by directly solving the complex vector equations. ${ }^{34}$ The real and imaginary parts of $\alpha(\omega)$ represent the inductive and resistive responses of the $\mathrm{Cu}$ shell. The real part will be a negative number denoting the diamagnetic strength of the metal and is proportional to the penetration depth of the magnetic field, whereas the imaginary part is the eddy current loss decided by the penetration depth and the current strength, both of which vary with frequency. Under the quasistatic condition, the magnetic polarizability of the shell will approach a constant as the frequency increases and this constant would not change with the shell thickness when $t_{\mathrm{m}}$ is larger than the penetration depth. Such frequency-independent property will enable the FM outer shell to balance the inner diamagnetic response over a large bandwidth at high frequencies. This is verified from the curves (Figures $1 \mathrm{a}$ and $\mathrm{b}$ ), which show that this $\mathrm{Cu}$ shell will have the same magnetic polarizability as the thickest one (i.e., $t_{\mathrm{m}}=20 \mathrm{~mm}$; actually, in this case, it is a solid sphere) when $\delta$ is reduced to be smaller than $t_{\mathrm{m}}$ at high frequencies. It also indicates the existence of the 'cutoff' working frequency for different shells (as the diamagnetic inner component for a magnetic cloak). Of note is that the opposite dependences of damping strength and penetration depth on frequency lead to peaks appearing in the $\operatorname{Im}(\alpha)$ curves, which in practice should be shifted out of the frequency band of interest in the device design. For a usual near-field metal probe with working frequencies typically above $5 \mathrm{kHz}, t_{\mathrm{m}}=5 \mathrm{~mm}$ could be regarded as a lower limit after taking the eddy current loss into account. For the proof of principle and fabrication convenience, we used a $10-\mathrm{mm}$-thick $\mathrm{Cu}$ shell in our cloak device as a trade-off between size and bandwidth. It should be noted that the diamagnetic polarizability of this $\mathrm{Cu}$ shell 
a

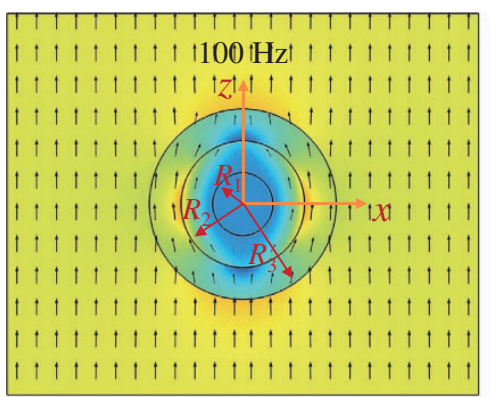

d

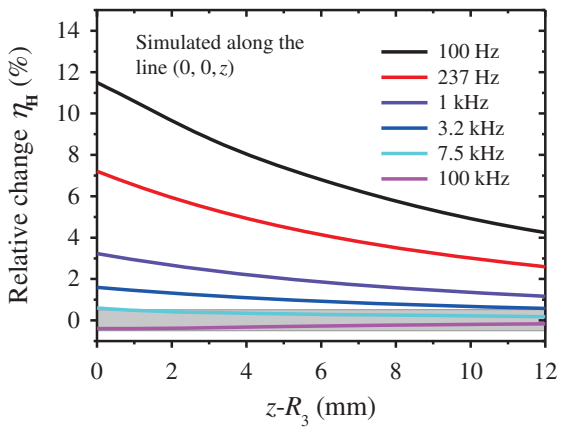

b

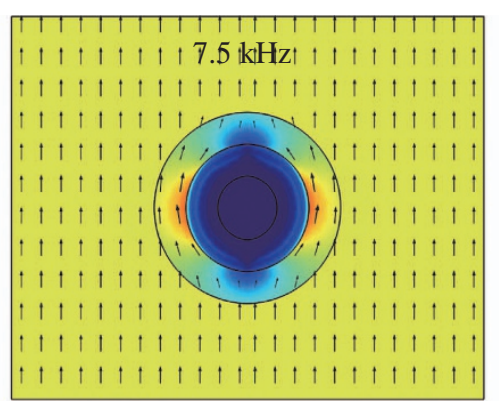

e

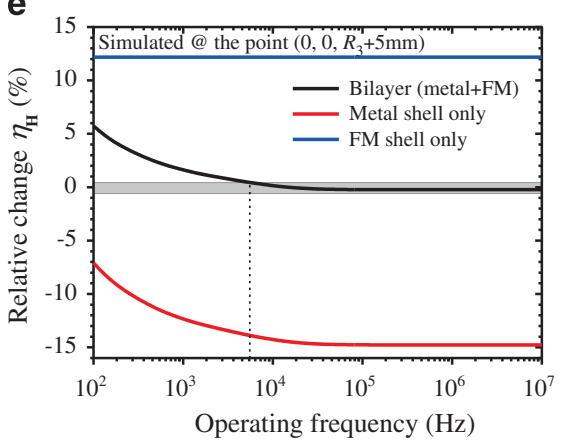

C

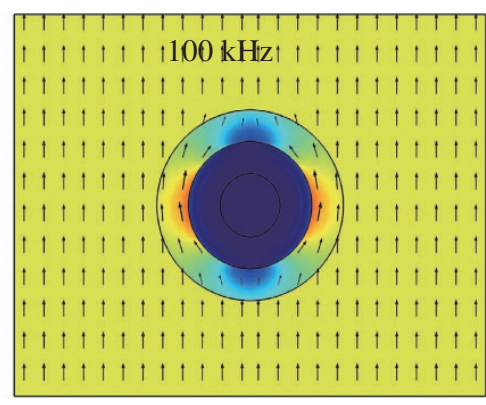

f

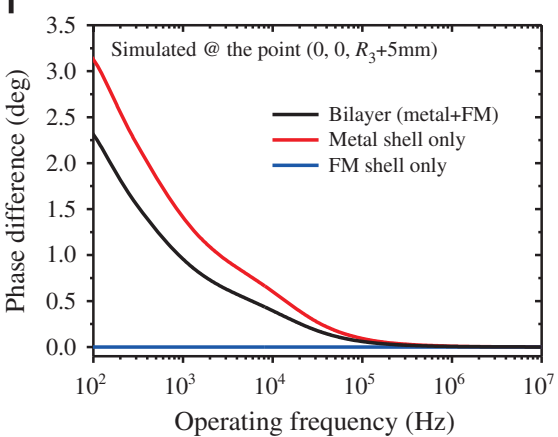

Figure 2 Simulated cloaking results. The magnetic field profiles at $100 \mathrm{~Hz}(\mathbf{a}), 7.5 \mathrm{kHz}$ (b) and $100 \mathrm{kHz}$ (c). Different colors denote the normalized field amplitudes at time $t=0$, and the arrows indicate the direction of the local field at the same moment. (d) Relative field change $\eta_{\mathbf{H}}$ along the $z$ axis at various frequencies. Relative field change $\eta_{\mathbf{H}}$ (e) and phase difference (f) at different operating frequencies for the bilayer sample and two references (metal or FM shell only) at $\left(0,0, R_{3}+5 \mathrm{~mm}\right)$. The gray shaded region in (e) profiles the range $\eta_{\mathbf{H}} \leqslant 0.5 \%$.

a

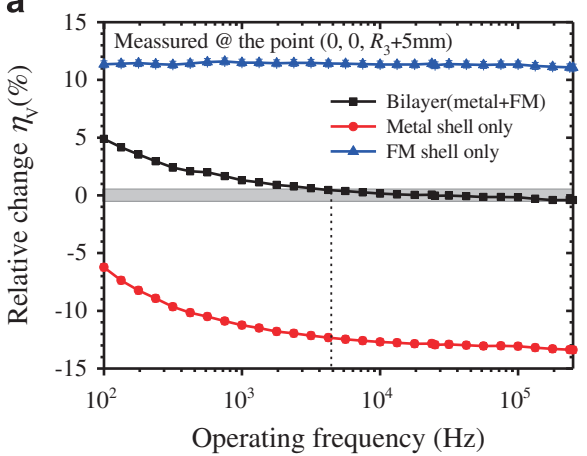

b

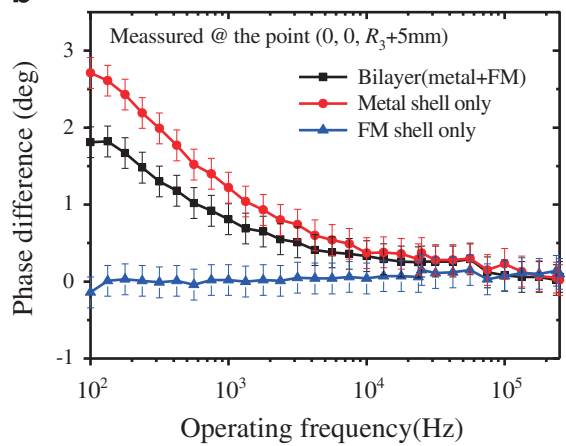

Figure 3 Measured cloaking results. Relative voltage change $\eta_{V}(\mathbf{a})$ and phase difference (b) for our cloaking sample and the two references (metal or FM shell only) from 100 to $250 \mathrm{kHz}$ at the point $\left(0,0, R_{3}+5 \mathrm{~mm}\right)$. The gray region in (a) corresponds to the area $\left|\eta_{H}\right| \leqslant 0.5 \%$. The measurement phase uncertainty in (b) is $0.3^{\circ}$.

unfavorably exhibits a slow variation from 10 to $10^{3} \mathrm{kHz}$ before saturation, which is unavoidable and inferior to the constant response of an SC. ${ }^{24}$ This inherent imperfection will limit the band performance of a metal-based magnetic cloak. In response, in this work we will define that a good cloaking effect is achieved if the absolute relative field change caused by the device is $<0.5 \%$ (the formula is given later). We also note that the above analysis is mainly for explaining why the metal shell can replace an SC shell at a relatively high frequency, but not in DC or very low frequency. In the following analysis, we need to use numerical simulation for the near-field effect of the magnetic cloaking.
For the outer FM shell, there is no simple equation to calculate the parameters when a permeable inner metal shell is used. Here we rely on computer simulation to optimize the magnetic and structural parameters for this layer with the input values given by $\mu_{\mathrm{FM}}=\frac{2 R_{3}^{3}+R_{2}^{3}}{2 R_{3}^{3}-2 R_{2}^{3}}$ (Equation (1) in ref. 24), and the conductivity remains zero $\left(\sigma_{\mathrm{FM}}=0\right)$, assuming an SC inner shell. Figures $2 \mathrm{a}-\mathrm{c}$ plot the magnetic field distributions of our optimized bilayer cloaking device at three frequencies, for example, $100,7.5$ and $100 \mathrm{kHz}$, respectively. Our cloak consists of a $10-\mathrm{mm}-$ thick metal shell and an FM shell of the same thickness (i.e., $R_{1}=R_{2} / 2=R_{3} / 3=10 \mathrm{~mm}$ and $\mu_{\mathrm{FM}}=1.62$ ) (Figure 2a) with a total diameter of $60 \mathrm{~mm}$. At $100 \mathrm{~Hz}$ (Figure 2a), a magnetic 
a

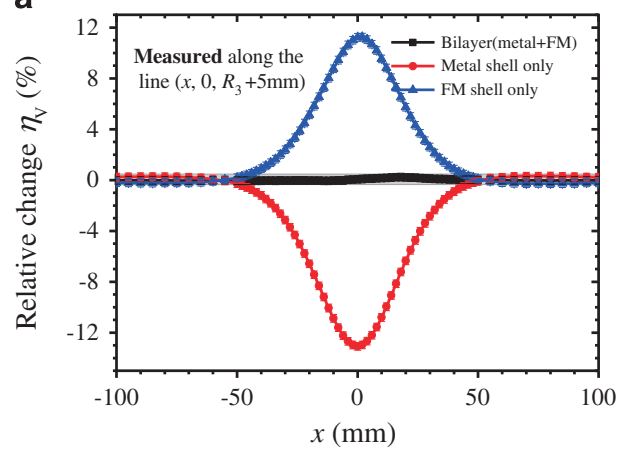

b

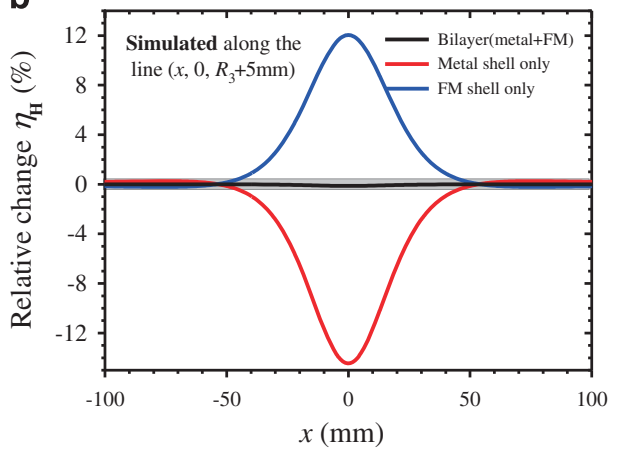

Figure 4 Near-field change. Measured (a) and simulated (b) relative changes of the nearby magnetic field for the bilayer sample and two references (metal or FM shell only) along the line $\left(x, 0, R_{3}+5 \mathrm{~mm}\right)$ with $x \in[-100 \mathrm{~mm}, 100 \mathrm{~mm}]$. The measured $\eta_{V}$ has an uncertainty of $0.3 \%$. The gray region corresponds to the area $\left|\eta_{V}\right| \leqslant 0.5 \%$. It is seen that the measured result could reproduce the simulation quite well.

a

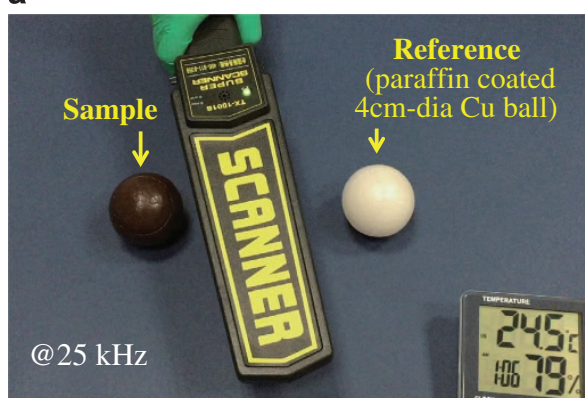

b

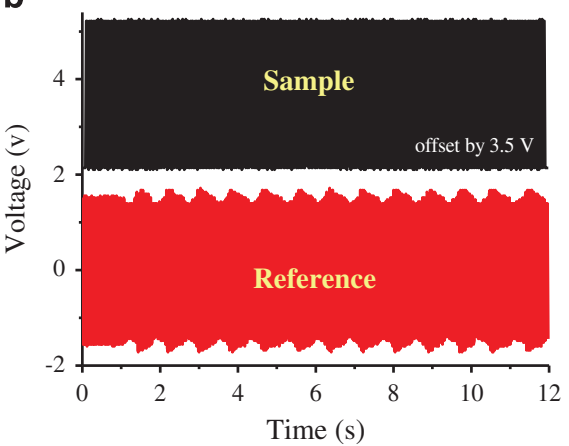

Figure 5 Characterization by a real metal scanner. (a) A picture of the measurement setup, including the bilayer sample and a reference (paraffin-coated 4-cm-diameter $\mathrm{Cu}$ ball). A temperature meter is placed in the lower right corner. (b) Measured voltage signals by an oscilloscope connected to the pick-up coil of the scanner. Modulation and invariance of the induced voltage signal are manifested when the scanner is swept over the reference and sample, respectively.

field will penetrate through the metal and enter the central air cavity, whereas it is shielded out at $7.5 \mathrm{kHz}$ (Figure 2b) and $100 \mathrm{kHz}$ (Figure 2c). The disturbance to the external incident field at the two latter frequencies is greatly minimized by this bilayer structure, which is quantitatively described by the variation curves of the magnetic field amplitude along the $z$ axis (Figure 2d). Here the relative field change is defined by $\eta_{H}=\left(|H|-\left|H_{0}\right|\right) /\left|H_{0}\right|$, with $H$ and $H_{0}$ equal to the magnetic fields with and without the sample. The criterion $\left|\eta_{H}\right|<0.5 \%$ is satisfied in the whole spatial range at 7.5 and $100 \mathrm{kHz}$. At the location $\left(0,0, R_{3}+5 \mathrm{~mm}\right)$, Figure $2 \mathrm{e}$ shows that the bilayer sample becomes a good cloaking device at $f>6 \mathrm{kHz}$. However, the metal shell only or the bilayer sample will indeed modify the phase value of the exciting field due to ohmic damping loss, which is obvious below $10^{5} \mathrm{~Hz}$ (Figure 2f). This is another compromise inherent in using a metal shell. However, the absolute phase difference for the bilayer sample is very small and $<1^{\circ}$ at above $1 \mathrm{kHz}$, which might not be serious in practice. In addition, metal detectors, including handheld ones, usually measure the field strength.

\section{Experimental verification}

Hereafter, we carry out an implementation of the proposed cloaking device by using paraffin-doped NiZn ferrite and copper as the outer and inner shells, respectively. The fabrication method is described elsewhere. ${ }^{24}$ The magnetic field is excited by a customdesigned Helmholtz coil fed by a signal generator and inductively measured by a lock-in amplifier via a small detection loop. The measurement frequency varies from 100 to $250 \mathrm{kHz}$, decided by our lock-in amplifier. The details on sample preparation and signal measurement can be found elsewhere. ${ }^{24}$ Figure $3 a$ plots the relative voltage change $\eta_{V}$ of the bilayer sample at various frequencies together with the references of pure metal and FM shells measured at $\left(0,0, R_{3}+5 \mathrm{~mm}\right)$. Here, $\eta_{V}=\left(|V|-\left|V_{0}\right|\right) /\left|V_{0}\right|$ is calculated by the measured induction voltages with $(V)$ and without $\left(V_{0}\right)$ the sample, which may have a slight difference from the simulated relative change of magnetic field $\eta_{\mathbf{H}}$ due to the spatial and angular averaging effects caused by the finite size of our detection loop (diameter $=4 \mathrm{~mm}$ ). It is seen that the combination of the $\mathrm{Cu}$ and FM shells can favorably balance out each other's influence at frequencies $>\sim 5 \mathrm{kHz}$, where $\left|\eta_{V}\right|<0.5 \%$, which is in good agreement with the numerical prediction (Figure 2e). The measured phase variation (Figure $3 \mathrm{~b}$ ) at the same location also matches the numerical predictions (Figure 2f) quite well. Phase variation due to the resistive attenuation is increased at $f<10 \mathrm{kHz}$. The operational band of a metal-based magnetic cloak would be narrowed if probed with a phase-sensitive appliance. 
Figure $4 \mathrm{a}$ plots the measured relative change $\eta_{V}$ along a line $\left(x, 0, R_{3}+5 \mathrm{~mm}\right)$ with $|x| \leqslant 100 \mathrm{~mm}$ for the bilayer sample and the two references at a typical frequency of $25 \mathrm{kHz}$. For the cloak, the measured $\eta_{V}$ is within our measurement uncertainty limit of $0.3 \%$, in quantitative agreement with the simulation (Figure 4b). The phase change of the magnetic field caused by the bilayer sample is also reduced to the level of our measurement resolution, that is, $0.2^{\circ}$ at $25 \mathrm{kHz}$. Since the sample is spherically symmetric, a similar level of field disturbance is expected in the whole space.

The above results indicate that a magnetic cloak could be engineered in the desired frequency bands using a combination of doped ferrite and metal shells. It is interesting that we propose to use metal to hide metal, but we must stress that the simple bilayer approach only works for cylindrical or spherical cloaking topologies; for other structures, the material parameters for the cloak will be very complex even under the quasistatic condition. Nevertheless, any metallic object of any shape could be concealed if placed inside the inner cavity of the spherical cloak by properly scaling the cloak size (actually the shape of the metallic object to be hidden does not matter once placed inside a spherical metal shell as long as the thickness of the metal shell is larger than the penetration depth). The ultimate cloaking performance will depend on the resolution of the probing device. Here we use a commercial handheld metal scanner to examine the actual performance of our device at a working frequency of $25 \mathrm{kHz}$. The alarm will be triggered if the probing magnetic field is distorted because of the existence of any metallic object. The scanner is swept over the sample and the reference (10-mm-thick paraffin coated $\mathrm{Cu}$ ball of diameter $=40 \mathrm{~mm}$ ) back and forth (Figure 5a). The reference obviously changes the EM environment and modulates the voltage signal induced by the pick-up coil of the scanner (Figure 5b). In comparison, the bilayer sample gives no obvious change to the induced signal, thus is seemingly transparent to the probing field. Supplementary Video S1 shows that the probe is successfully 'blinded' without being alarmed by the cloak (at room temperature, as the nearby thermometer indicates), whereas sharp 'beeping' voices and red flashes of the indicator are triggered by the reference. This indicates an anti-EM induction detection capability as good as the one previously achieved with the SC component. ${ }^{24}$

\section{DISCUSSION}

In summary, we have demonstrated the technical possibility of setting up a room temperature three-dimensional quasistatic magnetic cloak using the FM-metal bilayer approach. Strong diamagnetic response and finite penetration depth are the key properties allowing a normal metal to play the role of an SC in shielding magnetic fields. The operational band, in principle, could be designed accordingly by controlling the thickness of the shell, but the resistive loss will be the ultimate condition to affect the overall cloaking performance by causing energy loss and phase variation, especially at low frequencies. Our device as demonstrated here is able to work from $\sim 5 \mathrm{kHz}$ up to the maximum measurement frequency of $250 \mathrm{kHz}$ (which is thought to be further extendable) under both uniform and inhomogeneous external fields. ${ }^{23}$ This bilayer cloak will be more advantageous for hiding small objects and at frequencies above $10 \mathrm{kHz}$, although in principle one could scale the size. If multilayer structures similar to a voltage transformer are used, the eddy current induced by the alternative magnetic field will probably decrease, and consequently, the conduction loss will decrease and the cloak performance at lower frequencies will be significantly improved. A practical application based on the present results may be envisioned for scenarios where shielding magnetic fields without disturbing them is needed.

\section{CONFLICT OF INTEREST}

The authors declare no conflict of interest.

\section{ACKNOWLEDGEMENTS}

The authors are grateful to the partial support from NSFC (61271085 and 91233208), NSFC of Zhejiang province LR15F050001 and the Program of Zhejiang Leading Team of Science and Technology Innovation (2010R50007).

1 Pendry, J. B., Schurig, D. \& Smith, D. R. Controlling electromagnetic fields. Science 312, 1780-1782 (2006).

2 Leonhardt, U. Optical conformal mapping. Science 312, 1777-1780 (2006).

3 Schurig, D., Mock, J. J., Justice, B. J., Cummer, S. A., Pendry, J. B., Starr, A. F. \& Smith, D. R. Metamaterial electromagnetic cloak at microwave frequencies. Science 314, 977-980 (2006).

4 Cai, W., Chettiar, U. K., Kildishev, A. V. \& Shalaev, V. M. Optical cloaking with metamaterials. Nat. Photon. 1, 224-227 (2007).

5 Zhang, S., Xia, C. \& Fang, N. Broadband acoustic cloak for ultrasound waves. Phys. Rev. Lett. 106, 24301 (2011).

6 Stenger, N., Wilhelm, M. \& Wegener, M. Experiments on elastic cloaking in thin plates. Phys. Rev. Lett. 108, 14301 (2012).

7 Ma, Y., Lan, L., Jiang, W., Sun, F. \& He, S. A transient thermal cloak experimentally realized through a rescaled diffusion equation with anisotropic thermal diffusivity. NPG Asia Mater. 5, e73 (2013).

8 Li, J. \& Pendry, J. B. Hiding under the carpet: a new strategy for cloaking. Phys. Rev. Lett. 101, 203901 (2008).

9 Liu, R., Ji, C., Mock, J. J., Chin, J. Y., Cui, T. J. \& Smith, D. R. Broadband groundplane cloak. Science 323, 366-369 (2009).

10 Ergin, T., Stenger, N., Brenner, P., Pendry, J. B. \& Wegener, M. Threedimensional invisibility cloak at optical wavelengths. Science 328, 337-339 (2010).

$11 \mathrm{Ma}$, H. F. \& Cui, T. J. Three-dimensional broadband and broad-angle transformationoptics lens. Nat. Commun. 1, 124 (2010).

12 Wang, N., Ma, Y., Huang, R. \& Ong, C. K. Far field free-space measurement of three dimensional hole-in-Teflon invisibility cloak. Opt. Express. 21, 5941-5948 (2013).

13 Chen, X., Luo, Y., Zhang, J., Jiang, K., Pendry, J. B. \& Zhang, S. Macroscopic invisibility cloaking of visible light. Nat. Commun. 2, 176 (2011).

14 Zhang, B., Luo, Y., Liu, X. \& Barbastathis, G. Macroscopic invisibility cloak for visible light. Phys. Rev. Lett. 106, 33901 (2011).

$15 \mathrm{Ma}$, Y., Liu, Y., Lan, L., Wu, T., Jiang, W., Ong, C. K. \& He, S. First experimental demonstration of an isotropic electromagnetic cloak with strict conformal mapping. Scientific Rep. 3, 2182 (2013).

16 Wood, B. \& Pendry, J. B. Metamaterials at zero frequency. J. Phys. Condens. Matter 19, 76208 (2007).

17 Gömöry, F., Solovyov, M., Šouc, J., Navau, C., Prat-Camps, J. \& Sanchez, A. Experimental realization of a magnetic cloak. Science 335, 1466-1468 (2012).

18 Narayana, S. \& Sato, Y. DC magnetic cloak. Adv. Mater. 24, 71-74 (2012).

19 Yang, F., Mei, Z. L., Jin, T. Y. \& Cui, T. J. DC electric invisibility cloak. Phys. Rev. Lett. 109, 53902 (2012).

20 Souc, J., Solovyov, M., Gömöry, F., Prat-Camps, J., Navau, C. \& Sanchez, A. A quasistatic magnetic cloak. N. J. Phys. 15, 53019 (2013).

21 Prat-Camps, J., Sanchez, A. \& Navau, C. Superconductor-ferromagnetic metamaterials for magnetic cloaking and concentration. Supercond. Sci. Technol. 26, 74001 (2013)

22 Solovyov, M., Šouc, J. \& Gömöry, F. Magnetic cloak for low frequency AC magnetic field. IEEE Trans. Appl. Superconduct. 25, 1-5 (2015).

23 Prat-Camps, J., Navau, C. \& Sanchez, A. A magnetic wormhole. Scientific Rep. 5, 12488 (2015).

24 Zhu, J., Jiang, W., Liu, Y., Yin, G., Yuan, J., He, S. \& Ma, Y. Three-dimensional magnetic cloak working from d.c. to $250 \mathrm{kHz}$. Nat. Commun. 6, 8931 (2015).

25 Han, T., Bai, X., Gao, D., Thong, J. T. L., Li, B. \& Qiu, C.-W. Experimental demonstration of a bilayer thermal cloak. Phys. Rev. Lett. 112, 54302 (2014).

26 Xu, H., Shi, X., Gao, F., Sun, H. \& Zhang, B. Ultrathin three-dimensional thermal cloak. Phys. Rev. Lett. 112, 54301 (2014).

$27 \mathrm{Ma}$, Y., Liu, Y., Raza, M., Wang, Y. \& He, S. Experimental demonstration of a multiphysics cloak: manipulating heat flux and electric current simultaneously. Phys. Rev. Lett. 113, 205501 (2014).

28 Zeng, L. \& Song, R. Controlling chloride ions diffusion in concrete. Scientific Rep. 3, 3359 (2013). 
29 Schittny, R., Kadic, M., Bückmann, T. \& Wegener, M. Invisibility cloaking in a diffusive light scattering medium. Science 345, 427-429 (2014).

30 Raza, M., Liu, Y., Lee, E. H. \& Ma, Y. Transformation thermodynamics and heat cloaking: a review. J. Opt. 18, 44002 (2016).

31 Elyasi, M., Bhatia, C. S., Qiu, C.-W. \& Yang, H. Cloaking the magnons. Phys Rev. B 93, 104418 (2016).

32 Prat-Camps, J., Navau, C. \& Sanchez, A. Quasistatic metamaterials: magnetic coupling enhancement by effective space cancellation. Adv. Mater. 28, 4898-4903 (2016).

33 Bell, T., Barrow, B., Miller, J. \& Keiswetter, D. Time and frequency domain electromagnetic induction signatures of unexploded ordnance. Subsurf. Sens. Technol. Appl. 2, 153-175 (2001).

34 Wait, J. A conducting sphere in a time varying magnetic field. Geophysics 16, 666-672 (1951). (c) (i) This work is licensed under a Creative Commons Attribution 4.0 International License. The images or other third party material in this article are included in the article's Creative Commons license, unless indicated otherwise in the credit line; if the material is not included under the Creative Commons license, users will need to obtain permission from the license holder to reproduce the material. To view a copy of this license, visit http://creativecommons.org/licenses/by/4.0/

(C) The Author(s) 2017

Supplementary Information accompanies the paper on the NPG Asia Materials website (http://www.nature.com/am) 